\title{
Public Affairs-Management in Multinationalen Unternehmungen. Ergebnisse einer empirischen Untersuchung deutscher Unternehmungen in Rußland*
}

\author{
Dirk Holtbrügge, Nicola Berg ${ }^{* *}$
}

Der Beitrag beschäftigt sich mit einer detaillierten Analyse des Public Affairs Management in Multinationalen Unternehmungen. Insbesondere werden deutsche Unternehmen in Russland und dabei die Beziehungen zu ihren nicht marktlichen Interessengruppen untersucht. Begonnen wird mit der Darlegung der dem Beitrag zugrundeliegenden konzeptionellen und begrifflichen Grundlagen der Beziehungen von Multinationalen Unternehmungen zu ihren sozio-politischen Interessengruppen. Danach wird der Aufbau der empririschen Untersuchung aufgezeigt. Anschließend werden die zentralen Ergebnisse dieser Untersuchung präsentiert und kritisch diskutiert. Die Ausführungen schließen mit einer Gesamtbewertung und der Ableitung von Implikationen für die zukünftige Forschung.

This article deals with a detailed analysis of Public Affairs-Management in multi-national enterprises. Special focus is on German firms in Russia and their relationship to their non-market interest groups. The article sets out with a description of conceptional and terminological foundations in the area of the multi-national businesses' relations with their socio-political stakeholders. After explaining the setup of the empirical investigation, the authors present central results of the study, followed by a critical discussion. The paper closes with an overall evaluation and an outlook on possible implications for future research.

\footnotetext{
* manuscript received: 21.10.2000; accepted: 11.04.2001;

** Dirk Holtbrügge, geb. 1964, wissenschaftlicher Oberassistent am Lehrstuhl für Unternehmensführung, Universität Dortmund; Nicola Berg, geb. 1969, wissenschaftliche Mitarbeiterin am Lehrstuhl für Unternehmensführung, Universität Dortmund;
} 


\section{Bedeutung sozio-politischer Anliegen und Interessengruppen für die Tätigkeit Multinationaler Unternehmungen}

Im Zuge der Globalisierung der Wirtschaft setzt sich zunehmend die Erkenntnis durch, daß Multinationale Unternehmungen nicht nur mit marktlichen Interessengruppen wie Kunden, Lieferanten und Anteilseignern (Shareholder) interagieren, sondern in einem besonderen Maße auch dem Einfluß sozio-politischer Interessengruppen (Stakeholder) wie Regierungen, Medien und NichtRegierungsorganisationen ausgesetzt sind. Grenzüberschreitende Wertschöpfungsprozesse, Produktionsverlagerungen in Niedriglohnländer und Umsätze, die das Bruttoinlandsprodukt vieler Staaten übersteigen, sind nur einige der Ursachen, die zu einer erhöhten öffentlichen Aufmerksamkeit beitragen. Darüber hinaus setzt sich in vielen Ländern die Auffassung durch, daß die Tätigkeit von Multinationalen Unternehmungen vielfältige negative soziale und ökologische Effekte verursachen kann, die sich nur durch eine aktive politische Einflußnahme reduzieren lassen.

Entgegen dieser großen politischen, sozialen und ökologischen Bedeutung Multinationaler Unternehmungen liegen bislang nur vereinzelte Beiträge vor, die sich explizit den Beziehungen von Multinationalen Unternehmungen zu ihren nicht-marktlichen Interessengruppen widmen. Die meisten Untersuchungen im Bereich der internationalen Managementforschung sind weiterhin auf unternehmungsinterne Aspekte sowie auf die Beziehungen zu marktlichen Interessengruppen ausgerichtet. Dieses gilt insbesondere für den deutschsprachigen Raum.

Die vorliegende Untersuchung will einen Beitrag dazu leisten, diese Forschungslücke zu schließen und das Public Affairs Management in Multinationalen Unternehmungen einer detaillierteren Analyse zu unterziehen. Im zweiten Kapitel werden dazu zunächst einige wichtige konzeptionelle und begriffliche Grundlagen der Beziehungen von Multinationalen Unternehmungen $\mathrm{zu}$ ihren sozio-politischen Interessengruppen herausgearbeitet und der Aufbau einer empirischen Untersuchung von deutschen Multinationalen Unternehmungen in Rußland dargestellt. Anschließend werden die zentralen Ergebnisse dieser Untersuchung präsentiert und kritisch diskutiert. Die Ausführungen schließen mit einer Gesamtbewertung und der Ableitung von Implikationen für die zukünftige Forschung.

\section{Aufbau und Ablauf der empirischen Studie}

Das Ziel der Untersuchung besteht darin zu erforschen, wie das Verhalten Multinationaler Unternehmungen durch unterschiedliche sozio-politische Interessengruppen beeinflußt wird und welche Strategien, Instrumente und Organisationsformen des Public Affairs Management Multinationale 
Unternehmungen entwickeln, um mit den von diesen erhobenen Ansprüchen umzugehen. Zur Realisierung dieser Zielsetzung wurde eine empirische Untersuchung unter Tochtergesellschaften deutscher Multinationaler Unternehmungen in Rußland durchgeführt. ${ }^{3}$

Als Untersuchungseinheiten wurden 14 Tochtergesellschaften deutscher Multinationaler Unternehmungen aus der chemischen Industrie, der pharmazeutischen Industrie, dem Anlagenbau und der Automobilindustrie ausgewählt. Die untersuchten Unternehmungen gehören zu den größten deutschen Investoren in Rußland und beschäftigen durchschnittlich rund 250 Mitarbeiter.

Die Datenerhebung erfolgte in Form persönlicher Interviews mit Hilfe eines halb-standardisierten Fragebogens während eines Forschungsaufenthalts im Juli 2000 in Moskau. ${ }^{4}$ Gesprächspartner waren i.d.R. der höchstrangige Stammhausdelegierte. Trotz des hohen Zeit- und Kostenaufwands erschien eine persönliche Befragung notwendig, da im Rahmen der Untersuchung zahlreiche sensible Themengebiete wie z.B. Konfliktsituationen mit Interessengruppen, Lobbying oder der Einsatz von Bestechungsgeldern zur Durchsetzung bestimmter Interessen angesprochen werden. Eine Auskunftsbereitschaft zu diesen Aspekten kann i.d.R. erst nach dem Aufbau eines gewissen Vertrauensverhältnisses vorausgesetzt werden, das wiederum nur durch eine persönliche Interaktion mit den Befragten möglich ist.

Die theoretische Grundlage der Untersuchung bildet der Stakeholder-Ansatz. Dieser basiert auf der Annahme, daß der Erfolg einer Unternehmung nicht nur auf der effizienten Koordination und Kontrolle ihrer internen Aktivitäten sowie ihrer marktlichen Austauschprozesse beruht. Eine Unternehmung wird vielmehr als Fokus von Interessengruppen verstanden, die an die Unternehmung unterschiedliche Anliegen herantragen und ihre Aktivitäten durch unterschiedliche Maßnahmen beeinflussen (vgl. Freeman 1984). Der Erfolg einer Unternehmung hängt damit von deren Fähigkeit $a b$, für ihr Verhalten die Legitimation von allen Interessengruppen zu erhalten, auf die diese bei der Realisierung ihrer Ziele und Handlungen angewiesen ist (vgl. Kostova/Zaheer 1999, S. 64).

3 Die Untersuchung des Public Affairs Management in Tochtergesellschaften deutscher Multinationaler Unternehmungen in Rußland ist Teil einer von der VolkswagenStiftung finanziell geförderten umfassenden empirischen Untersuchung in Deutschland, Frankreich, Rußland, Indien, China und den USA. Zu ersten Ergebnissen der Studie vgl. Welge/Berg (1999) und Berg/Holtbrügge (2001) für Indien und Berg/Holtbrügge (2000) für China.

${ }^{4}$ Moskau stellt neben St. Petersburg die wichtigsten Industriemetropole des Landes dar und weist die mit Abstand höchste Zahl von Tochtergesellschaften ausländischer Unternehmungen auf. 
$\mathrm{Zu}$ dieser Legitimitätsstiftung stehen einer Unternehmung unterschiedliche Strategien und Instrumente zur Verfügung, die unter dem Begriff des Public Affairs Management zusammengefaßt werden können. Unter Public Affairs Management verstehen Welge/ Holtbrügge (1998, S. 263) "die Gestaltung der Beziehungen zu den externen sozio-politischen Interessengruppen einer Unternehmung. Dessen Ziel ist es, potentielle, latente und manifeste Konflikte mit diesen Interessengruppen effizient zu handhaben sowie Wettbewerbsvorteile, die sich durch die Berücksichtigung oder Beeinflussung sozio-politischer Anliegen eröffnen, möglichst weitgehend auszuschöpfen". Die Instrumente, die Multinationale Unternehmungen einsetzen, um ihre Beziehungen $\mathrm{zu}$ ihren externen sozio-politischen Interessengruppen möglichst vorteilhaft zu gestalten, stellen den zweiten Aspekt des konzeptionellen Bezugsrahmens dar.

\section{Sozio-politische Interessengruppen von Tochtergesellschaften deutscher Multinationaler Unternehmungen in Rußland}

$\mathrm{Zu}$ Beginn der Interviews wurden die Befragten darum gebeten, anhand einer siebenstufigen Likertskala die Bedeutung unterschiedlicher sozio-politischer Interessengruppen zu beurteilen, die sich restriktiv oder unterstützend auf ihre unternehmungspolitischen Entscheidungen auswirken. Übereinstimmend wurde dabei die russische Gastlandregierung als besonders wichtig eingestuft. An zweiter Stelle steht die jeweilige regionale Administration, gefolgt von der Mafia und wichtigen Verbänden. Eine mittlere Bedeutung kommt den Medien und der entsprechenden lokalen Administration zu, während Bürgerinitiativen, Gewerkschaften und andere Interessengruppen keinen Einfluß auf die Tätigkeit ausländischer Unternehmungen haben. Gleiches gilt für internationale Organisationen. Einen Überblick über die Bedeutung unterschiedlicher soziopolitischer Interessengruppen gibt Abbildung 1 wieder.

Die zentrale Bedeutung der russischen Regierung und der ihr angegliederten Ministerien und Komitees wird vor allem mit der hohen Regulierungsdichte begründet, die in Rußland trotz des eingeleiteten Übergangs zur Marktwirtschaft immer noch vorherrscht. Insbesondere Genehmigungsverfahren sowie die Kontakte zu den russischen Steuer- und Zollbehörden werden als sehr aufwendig und langwierig beurteilt. Die Gründe dafür sind eine ausufernde Bürokratie, intransparente Entscheidungsstrukturen und die weitverbreitete Korruption. Die zahlreichen Regierungsumbildungen seit 1991 haben zudem zu einem häufigen Wechsel der zentralen Entscheidungsträger sowie zu einer allgemeinen Verunsicherung der Staatsbediensteten geführt. 
Abb. 1: Bedeutung unterschiedlicher sozio-politischer Interessengruppen aus der Sicht der Befragten

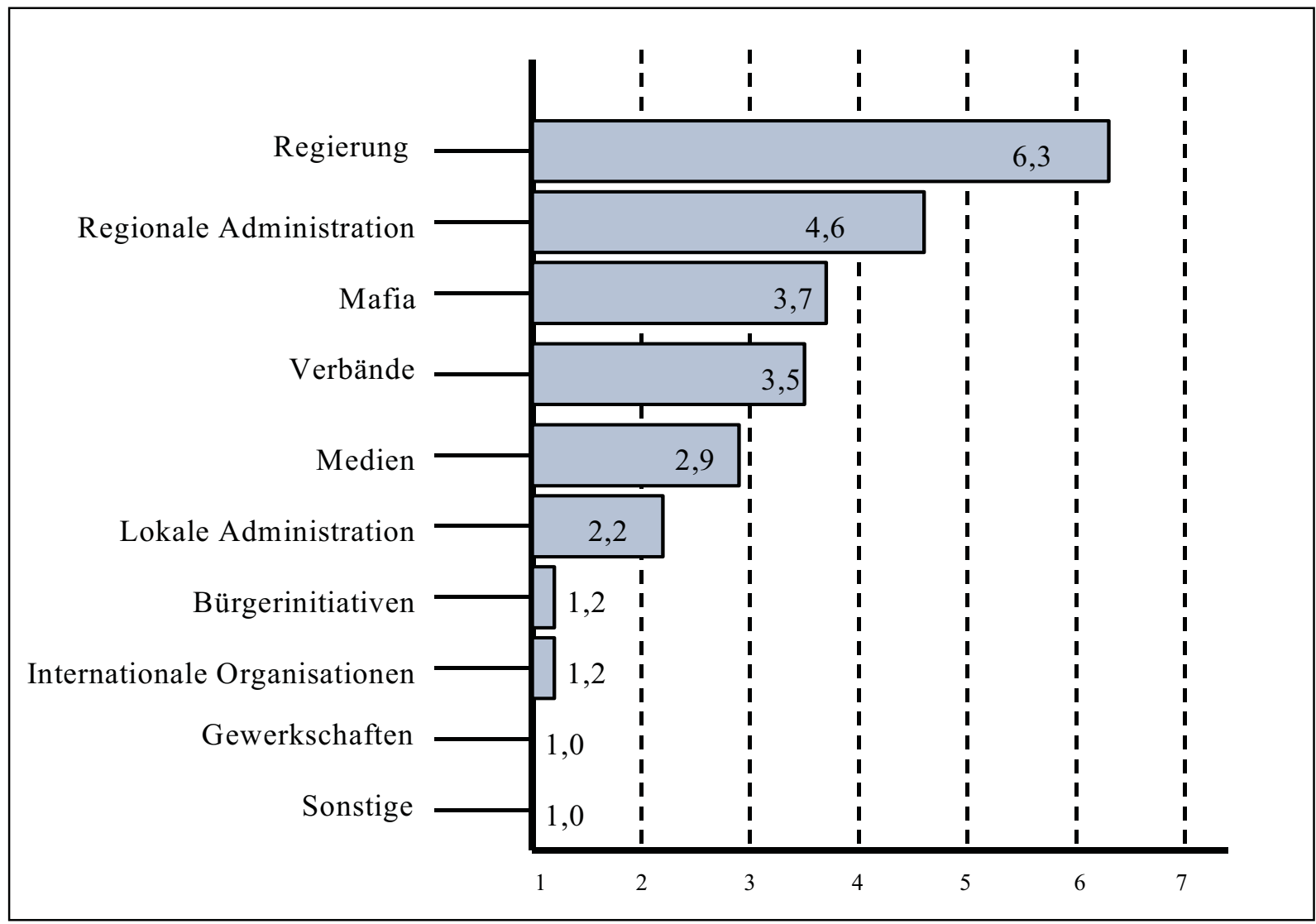

\subsection{Gastlandregierung}

Ein weiteres Problemfeld stellt nach Auskunft der Befragten die geringe Rechtssicherheit dar. Zwar wird positiv bewertet, daß neu verabschiedete Gesetze inzwischen sehr schnell zugänglich sind, diese werden jedoch von den zuständigen Behörden im konkreten Einzelfall oft uneinheitlich, widersprüchlich und teilweise willkürlich ausgelegt. In der Vergangenheit wurden gesetzliche Bestimmungen zudem häufig rückwirkend geändert, so daß die Planungssicherheit gering ist. Rechtskenntnis ist in Rußland deshalb zwar sehr wichtig, reicht aber allein noch nicht aus. Einer der Befragten bemerkte deshalb: "In Rußland sind nicht Gesetze, sondern persönliche Beziehungen wichtig. Das wichtigste Recht ist das Telefonrecht, d.h. zu einem einflußreichen Entscheidungsträger durchgestellt zu werden, der das Problem durch Anweisung regelt."

Die Mehrheit der Befragten erwartet, daß die durch den neuen russischen Präsidenten Vladimir Putin eingeleiteten Maßnahmen die Rechtssicherheit und Transparenz von Entscheidungen mittelfristig erhöhen werden. Diese Einschätzung muß jedoch eher kritisch beurteilt werden, da die von Putin proklamierte "Diktatur des Gesetzes" in vielen Bereichen weniger auf die Ver- 
wirklichung von Rechtssicherheit in einem westlichen Sinne als vielmehr auf eine "Entwicklungsdiktatur" (etwa nach chilenischem oder südkoreanischen Vorbild) abzielt, bei der die Verankerung von Demokratie und Bürgerrechten kompromißlos macht- und wirtschaftspolitischen Zielen untergeordnet wird. Kritiker Putins wenden deshalb ein, daß die Zentralisierung der politischen Macht langfristig zu einer Lähmung der gerade erst aufkommenden wirtschaftlichen Initiative führen wird und deshalb nicht im strategischen Interesse ausländischer Investoren liegen kann. Vor dem Hintergrund der Globalisierung wird darüber hinaus bezweifelt, ob verstärkte staatliche Eingriffe in die russische Volkswirtschaft höhere Wachstumseffekte als eine konsequente wirtschaftliche Liberalisierung bewirken werden (vgl. etwa Seiffert 2000, S. 97). Vor allem aber wird das Vorgehen Putins in Tschetschenien kritisiert und auf den Einfluß verwiesen, den der dort geführte Krieg auch auf das Investitionsrisiko in Rußland habe (vgl. z.B. Glucksmann 2000).

\subsection{Regionale und lokale Administration}

Erschwert wird der Umgang mit politischen Entscheidungsträgern auch durch die unübersichtlichen Machtverhältnisse zwischen der Zentralregierung und der jeweiligen regionalen und lokalen Administration. ${ }^{5}$ Rußland besteht aus insgesamt 80 Föderationssubjekten, die über eine eigenständige Investitions-, Steuer- und Arbeitsgesetzgebung verfügen. ${ }^{6}$ Deren politische Bedeutung ist vor allem während der Amtszeit des früheren Präsidenten Boris Jelzin stark angestiegen, der den Vertretern der Regionen im Föderationsrat, dem Oberhaus des Russischen Parlaments, als Ausgleich für deren Zustimmung zu wichtigen Gesetzen eine hohe Autonomie eingeräumt hatte. Nur dadurch war es ihm möglich, ein Gegenwicht zu der von kommunistischen und nationalistischen Abgeordneten dominierten Staatsduma, dem Unterhaus des Russischen Parlaments, zu schaffen.

Seit den Dumawahlen im Dezember 1999 und der Wahl des neuen russischen Präsidenten Vladimir Putin im März 2000 zeichnet sich jedoch eine verstärkte Zentralisierung der politischen Macht ab (vgl. Šejnis 2000). Ein wichtiges Vorhaben Putins besteht darin, die Macht der Regionen zu begrenzen und diese in einem stärkeren Maße als bisher auf die Umsetzung der zentral verabschiedeten Gesetze und Verordnungen zu verpflichten. Die Auswirkungen dieses Vorhabens auf ausländische Unternehmungen sind ambivalent. Während

5 Zum Verhältnis zwischen föderaler und regionaler Gesetzgebung vgl. ausführlich Bell (1999).

6 Die einzelnen Regionen Rußlands weisen nicht nur in politischer, sondern auch in geographischer, klimatischer, wirtschaftlicher, religiöser und ethischer Hinsicht eine äußerst heterogene Struktur auf (vgl. dazu ausführlich Kappeler 1992; Götz/Halbach 1994). 
dies einerseits zu einer Vereinheitlichung der politischen, ökonomischen und rechtlichen Rahmenbedingungen in den einzelnen Regionen führt, wird andererseits deren Wettbewerb um ausländische Investoren beschränkt. Langfristig dürfte dies vor allem für diejenigen ausländischen Unternehmungen $\mathrm{zu}$ Nachteilen führen, deren politisches und ökonomisches Gewicht zu gering ist, um eine starke Verhandlungsposition gegenüber der Zentralregierung zu erlangen.

\subsection{Mafia}

Neben den zentralen und regionalen politischen Machtorganen wird von den Befragten vor allem der russischen Mafia eine große Bedeutung für ihre Geschäftspolitik zugewiesen. Dieses Ergebnis scheint auf den ersten Blick den Eindruck zu bestätigen, den viele westliche Medien sowie zahlreiche in den letzten Jahren erschienene populärwissenschaftliche und dokumentarische Veröffentlichungen vermitteln. Dabei muß jedoch berücksichtigt werden, daß der Begriff "Mafia" in der russischen Öffentlichkeit vielfältig und in einem sehr weiten Sinne verwendet wird. Grob können dabei drei unterschiedliche Gruppen unterschieden werden:

Zur ersten Gruppe zählen Personen, die häufig bereits Ende der achtziger Jahre die sich eröffnenden Möglichkeiten privatwirtschaftlicher Tätigkeit ergriffen und etwa durch den Handel mit defizitären Gütern hohe Gewinne erzielt haben. Diese wurden häufig reinvestiert und zum Aufbau von inzwischen bedeutenden Unternehmungen investiert. Die Gleichsetzung dieser Unternehmer mit mafiösen Strukturen verweist damit eher auf eine - vor dem Hintergrund einer sehr egalitären Gesellschaft - als unsozial und unmoralisch empfundene Geschäftstüchtigkeit als auf kriminelle oder gar gewalttätige Tätigkeiten.

Die zweite Gruppe bilden organisierte illegale Strukturen, die vielfach mit der staatlichen Administration verwoben sind und deshalb als kryša (russ.: "Dach", besser: "Schutzschirm") bezeichnet werden. Dieser Gruppe zahlt die Mehrheit der untersuchten Unternehmungen eine Art "Schutzgeld", für das sie eine Gegenleistung erhalten, die nach deutschem Verständnis durch staatliche Strukturen erbracht würde. So beinhaltet nach Auffassung eines Befragten etwa die im Vergleich zum allgemeinen Preisniveau sehr hohe Miete, die ausländische Unternehmungen für Büros oder Wohnungen entrichten, neben der technischen Instandhaltung auch den Schutz vor Gewalt gegen Personen oder Sachen. Die Mafia wird in diesem Sinne weniger als Verbrecherorganisation, sondern vielmehr als "privater Sicherheitsdienst" verstanden, für dessen Dienstleistungen die Unternehmungen einen bestimmten Betrag entrichten, um nicht auf die Hilfe der in weiten Bereichen als hilflos empfundenen Miliz angewiesen zu sein.

Die dritte Gruppe bilden schließlich kriminelle Banden (bandity), die zumeist nach ethnischen Kriterien organisiert sind und in relativ festgelegten Regionen 
in Form von Schutzgelderpressungen, Entführungen und Inkassogeschäften bis hin zu Auftragsmorden tätig sind (vgl. dazu ausführlich Schlott 1996). Mit dieser Gruppe haben die untersuchten Unternehmungen nach eigener Auskunft nur wenig Kontakt, wobei es fraglich erscheint, ob die Befragten zu diesem Aspekt offen und ehrlich geantwortet haben. So wurden zwar stets eigene Kontakte verneint, mehrfach jedoch von anderen Managern erzählt, die auf diesem Gebiet unliebsame persönliche Erfahrungen gemacht haben.

Insgesamt hat nach Aussage der Befragten in den letzten Jahren der Einfluß der Mafia generell sowie vor allem das Ausmaß der offenen Gewalt in der Wirtschaft abgenommen. Dies bedeutet jedoch nicht zwangsläufig, daß auch die Kriminalisierung der Wirtschaft zurückgegangen ist. In vielen Fällen ist vielmehr eine "Routinisierung der Gewalt" und deren "Transformation in normale Geschäftspraktiken" zu beobachten (Radaev 1999). An die Stelle des spontanen Racketeering rücken dabei nachhaltigere und zivilisiertere Formen der Kontrolle von Unternehmungen durch organisierte kriminelle Strukturen. "Moskva banditskaya is becoming Moskva biznesmenskaya" (Elder 1999, S. 84).

\subsection{Verbände}

Für die untersuchten Unternehmungen stellen insbesondere die jeweiligen internationalen Branchenverbände wichtige Interessengruppen dar. Diese haben z.B. Einfluß auf die Festlegung von Normen und Standards, auf die Zertifizierung von Produkten sowie auf die Verabschiedung von branchenbezogenen Gesetzen und Verordnungen. Ähnliches gilt für die Interessenverbände ausländischer Unternehmungen wie den Verband der deutschen Wirtschaft in der Russischen Föderation, den European Business Club oder die American Chamber of Commerce, deren Vertreter etwa zu Konsultationen in russische Industrieministerien und -komitees eingeladen werden.

Daneben existieren noch zahlreiche russische Industrieverbände und -assoziationen, deren politischer Einfluß nach Auskunft der Befragten jedoch - mit Ausnahme des "Russischen Verbands der Industriellen und Unternehmer" unter der Leitung von Arkadij Vol'skij - als gering eingeschätzt wird. Für die Tätigkeit ausländischer Unternehmungen sind diese deshalb weder als Interessengruppen noch als Lobbyisten relevant.

\subsection{Medien}

Den Medien wird von den untersuchten Unternehmungen eine mittlere Bedeutung für ihre Tätigkeit zugemessen, wobei deren inhaltliche Bewertung kontrovers ausfällt. Insbesondere Befragte, die über gute russische Sprach- und Landeskenntnisse verfügen, schätzen die russischen Zeitungen und das Fernsehen als wichtige Kontrollorgane der russischen Regierung und 
einflußreicher Unternehmer ein und bescheinigen diesen eine kritische und mutige Haltung bei der Behandlung von strittigen und kontroversen Themen. Der Umgang mit diesen wird dabei als unkompliziert und fair empfunden.

Demgegenüber wird den Medien von anderen Befragten eine wenig objektive Berichterstattung vorgeworfen. Diese sind etwa der Ansicht, daß Probleme häufig übertrieben, über positive Entwicklungen dagegen kaum berichtet würde. Auch den meisten ausländischen Zeitungen und Fernsehsendern wird vorgeworfen, zu kritisch und negativ über Rußland zu berichten. "Wenn ich mir gelegentlich Reportagen auf SAT 1 oder RTL ansehe", so einer der Befragten, "bekomme ich den Eindruck, Rußland besteht nur aus bettelnden Kindern, Sexorgien bei Neureichen und brennenden Ölpipelines in Sibirien. Mit meinem Leben hier hat das nur sehr wenig zu tun." Die als reißerisch empfundenen Reportagen verzerrten deshalb nicht nur das Bild, das man im Ausland über Rußland gewinne, sondern mache es auch immer schwieriger, qualifizierte deutsche Mitarbeiter für eine Tätigkeit in Rußland zu gewinnen.

\subsection{Bürgerinitiativen}

Weitgehend ohne Einfluß auf die Tätigkeit ausländischer Unternehmungen sind dagegen russische Bürgerinitiativen und Nicht-Regierungsorganisationen (NGOs). Dies ist insofern überraschend, als die noch bis zu Beginn der neunziger Jahre zu beobachtende Lethargie und Passivität der Bevölkerung spürbar zurückgegangen ist. So stellt etwa Apressyan (1997, S. 1566) die zunehmende Eigeninitiative von Menschen und Familien als eines der wichtigsten Ergebnisse des Transformationsprozesses in Rußland heraus. Während der Staat früher fast alle Belange der Bürger bis ins Privatleben hinein geregelt hat, nehmen diese ihr Schicksal nun vermehrt selbst in die Hand. Dies gilt jedoch bislang fast ausschließlich nur für den privaten Bereich. Bürgerinitiativen und lokale Interessenvereinigungen sind dagegen immer noch stark unterentwickelt. Zwar gibt es in Rußland inzwischen Niederlassungen internationaler NGOs (wie z.B. Amnesty International, Greenpeace oder den World Wildlife Fund for Nature) und einzelne Initiativen wie z.B. das Helsinki Komitee für Menschenrechte oder die Gruppe Memorial, die sich für die Opfer der Stalin-Diktatur engagiert. Deren Einfluß im Bereich des Umweltschutzes, der Kommunalpolitik oder der Sozial- und Bildungspolitik ist jedoch insgesamt gering.

Überraschend ist, daß dies von einigen Befragten explizit bedauert wird. Bürgerinitiativen würden nach deren Auffassung nicht nur ein wünschenswertes Gegengewicht zum hohen Einfluß des Staates bilden, sondern auch die wirtschaftliche Dynamik des Landes fördern. Eine der untersuchten Unternehmungen unterstützt deshalb im Rahmen ihrer Sponsoringaktivitäten explizit auch die Tätigkeit von russischen NGOs, wenn auch im Vergleich zu anderen Aktivitäten nur in einem sehr geringen Umfang. 


\subsection{Gewerkschaften}

Ähnlich gering wie der Einfluß von Bürgerinitiativen ist auch die Bedeutung der Gewerkschaften. Zwar schreibt das russische Arbeitsrecht vor, daß die Geschäftsleitung von Unternehmungen mit dem jeweiligen betrieblichen Gewerkschaftskomitee Kollektivverträge abschließt, in denen z.B. die Basislöhne, Höhe und Bedingungen von Zusatzgratifikationen, Arbeitszeit und Ruheperioden, Arbeitsschutz, innerbetriebliche Weiterbildung, Disziplinarmaßnahmen gegen Mitarbeiter oder die Einrichtung und Nutzung betrieblicher Sozialeinrichtungen geregelt werden, für die Praxis ausländischer Unternehmungen ist diese Bestimmung jedoch weitgehend irrelevant (vgl. auch Holtbrügge 1995, S. 146 ff.).

Die geringe faktische Bedeutung der Gewerkschaften als Interessenvertretung der Arbeitnehmer kann vor allem darauf zurückgeführt werden, daß die Nachfolgeorganisationen der Staatsgewerkschaften wegen ihrer früheren engen $\mathrm{Zu}$ sammenarbeit mit den ehemaligen kommunistischen Führungen ("Transmissionsriemen der Partei") in eine tiefe Legitimations- und Identitätskrise geraten und gegenwärtig mit einem erheblichen Mitgliederschwund konfrontiert sind. Die häufig aus Bürgerbewegungen, Streikkomitees und neuen Formen der Selbstorganisation hervorgegangenen unabhängigen Gewerkschaften sind dagegen noch weitgehend mit innerorganisatorischen und finanziellen Problemen sowie der Anwerbung von Mitgliedern befaßt. Deren faktische Einflußund Mitbestimmungsmöglichkeiten werden zudem durch das Neben- und Gegeneinander verschiedener Industrie- und Betriebsgewerkschaften erheblich eingeschränkt.

\subsection{Internationale Organisationen}

Angesichts der geringen Einbindung Rußlands in die internationale Arbeitsteilung ist schließlich auch die Relevanz internationaler Organisationen aus Sicht der Befragten vernachlässigbar. Ausnahmen bilden lediglich Unternehmungen, die sich an Ausschreibungen internationaler Finanzorganisationen wie des Internationalen Währungsfonds, der Weltbank oder der Europäischen Bank für Wiederaufbau und Entwicklung beteiligen. Darüber hinaus könnte der Einfluß der deutschen Bundesregierung zunehmen, wenn diese - wie auf dem letzten G8-Gipfel im Juli 2000 in Okinawa von Bundeskanzler Schröder in Aussicht gestellt - wieder Hermes-Bürgschaften für Geschäfte deutscher Unternehmungen mit Rußland übernehmen würde. Insgesamt ist der Einfluß sozio-politischer Interessengruppen jedoch fast ausschließlich auf russische Akteure begrenzt. 


\section{Sozio-politische Anliegen an Tochtergesellschaften deutscher Multinationaler Unternehmungen in Rußland}

Faßt man die von den im vergangenen Abschnitt dargestellten Interessengruppen an die untersuchten Unternehmungen herangetragenen Anliegen zusammen, so fällt auf, daß dabei politische und ökonomische Anliegen eindeutig im Vordergrund stehen, während gesellschaftliche und insbesondere ökologische Anliegen als weitgehend irrelevant empfunden werden (vgl. Abb. 2). Dies kann auf die Funktion ausländischer Unternehmungen für die Transformation der russischen Wirtschaft zurückgeführt werden.

Abb. 2: $\quad$ Bedeutung unterschiedlicher Anliegen aus der Sicht der Befragten

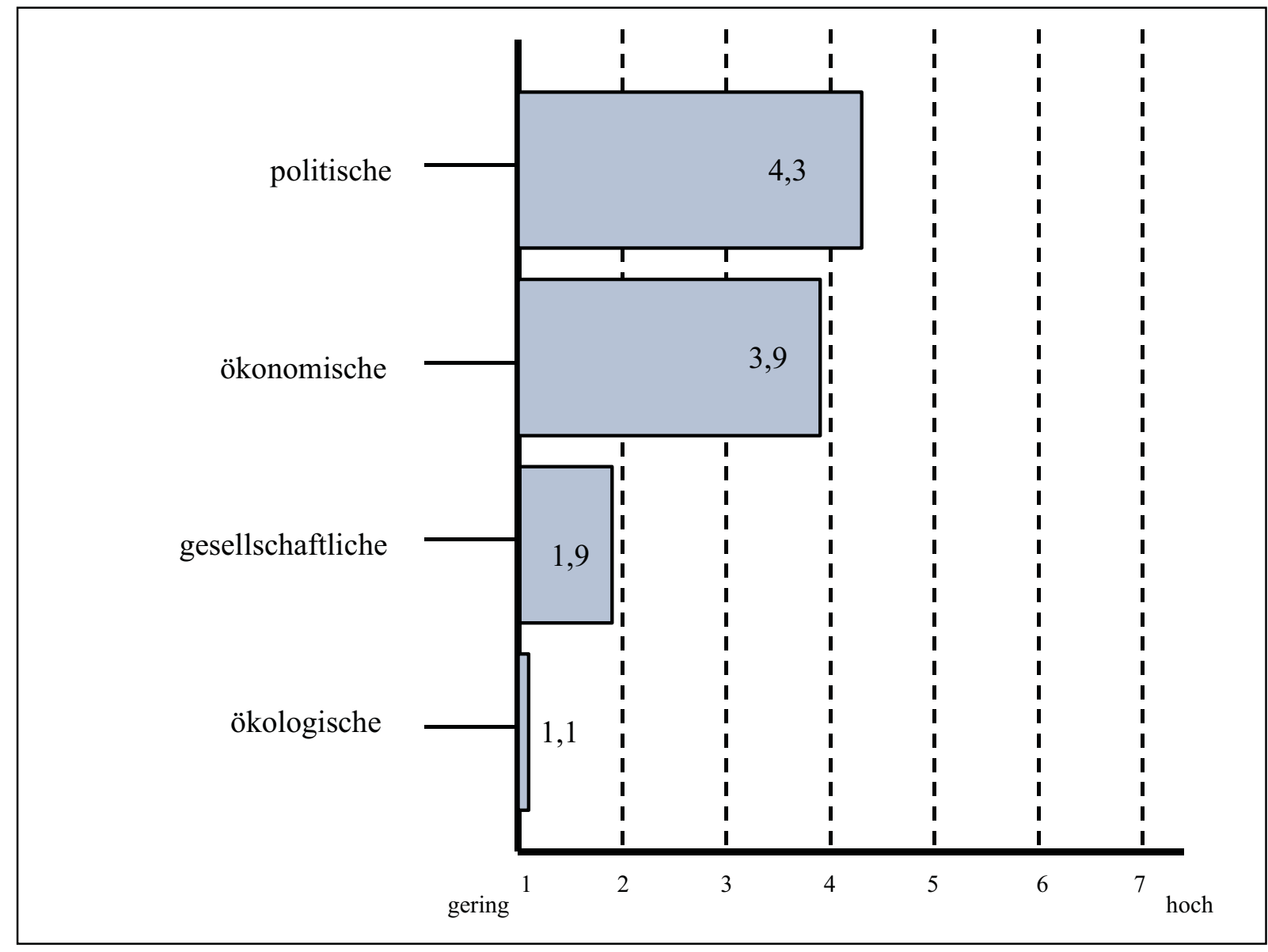

Stellten ausländische Direktinvestitionen nach ihrer Zulassung im Jahre 1987 zunächst nur "privatkapitalistische Inseln" (Holtbrügge 1989) innerhalb eines weiterhin zentralgeplanten Wirtschaftssystems dar, so fällt ihnen spätestens seit Mitte der neunziger Jahre eine Schlüsselrolle im Rahmen des Transformationsprozesses zu. Diese sollen durch den Import von Kapital, modernen Technologien und westlichem Management-Know-how nicht nur die internationale Wettbewerbsfähigkeit russischer Produkte und Dienstleistungen steigern, sondern durch die Verbesserung der Versorgungslage auch zur sozialen Flankierung des Transformationsprozesses beitragen und durch die Aus- 
übung von Wettbewerbsimpulsen auf russische Unternehmen bei der strukturellen Umgestaltung der Wirtschaft mitwirken. Als Katalysatoren und change agents des Transformationsprozesses kommt ausländischen Unternehmungen damit eine wichtige gesamtwirtschaftliche und politische Funktion zu (vgl. Holtbrügge 1996).

Auch viele Gouverneure verbinden mit der Tätigkeit ausländischer Unternehmungen in ihrer Region explizit ökonomische und politische Anliegen. Diese tragen zur Erhöhung der Steuereinnahmen bei und schaffen Arbeitsplätze und Qualifikationsmöglichkeiten. ${ }^{7}$ Viele Regionen werben deshalb mit spürbaren Investitionsanreizen um ausländische Investoren. Umgekehrt haben mehrere Befragte darauf hingewiesen, daß eine fehlende Unterstützung durch die lokale und regionale Administration zu erheblichen Schwierigkeiten führen oder die Tätigkeit ausländischer Unternehmungen sogar ganz gefährden kann.

Im Unterschied $\mathrm{zu}$ politischen und gesamtwirtschaftlichen Interessen sind soziale und ökologische Anliegen nach Auskunft der Befragten dagegen nur von geringer Relevanz. Überraschend ist, daß dabei etwa auch die Stiftungsinitiative der Bundesregierung "Erinnerung, Verantwortung und Zukunft" zur Entschädigung ehemaliger Zwangsarbeiter in deutschen Unternehmungen während des Nazi-Regimes keine große Bedeutung besitzt. Zwar haben das russische Fernsehen über die Gründung der Stiftung am 17. Juli 2000 berichtet und auch einige russische Tageszeitungen das Thema in kurzen Beiträgen aufgegriffen (vgl. z.B. Nezavisimaja gazeta, 19.7.2000, S. 6), die öffentliche Aufmerksamkeit ist jedoch sehr gering. So wurde nach Auskunft der Befragten keines der untersuchten Unternehmungen bislang konkret nach seiner Beteiligung an der Bundesstiftung gefragt. Zwar erhielten einzelne Unternehmungen Zuschriften von ehemaligen Zwangsarbeitern oder deren Rechtsanwälten, deren Zahl ist jedoch vor dem Hintergrund des betroffenen Personenkreises außerordentlich gering. Auch eine Aktion wie in den USA, wo jüdische Organisationen im Frühjahr 2000 in überregionalen Tageszeitungen Anzeigen mit Listen von Unternehmungen veröffentlicht haben, die während des NaziRegimes Zwangsarbeiter beschäftigt haben und sich noch nicht an der Stiftung beteiligt haben, wäre in Rußland nach übereinstimmender Einschätzung der Befragten nur sehr schwer vorstellbar bzw. ohne spürbaren Einfluß auf die öffentliche Meinung.

Neben der generellen Bedeutung unterschiedlicher sozio-politischer Anliegen wurden die Befragten um Auskunft darüber gebeten, ob ihre Unternehmung

\footnotetext{
${ }^{7}$ Nach Auskunft eines Vertreters der Delegation der Deutschen Wirtschaft in der Russischen Föderation machen ausländische Unternehmungen z.B. in einigen südrussischen Regionen trotz ihrer sehr geringen Zahl mehr als 50\% der regionalen Steuereinnahmen aus.
} 
Instrumente zur systematischen Identifikation und Analyse von Anliegen (z.B. in Form eines Environmental Scanning oder eines Issue Management) einsetzt. Dabei zeigte sich, daß keines der untersuchten Unternehmungen über ein solches Instrumentarium verfügt. Als Begründung dafür wurde angeführt, daß die hohe Dynamik der Umweltentwicklung eine solche systematische Analyse verhindert. Neue Issues kommen zumeist spontan auf und werden häufig bereits nach kurzer Zeit wieder durch andere Issues abgelöst. "Die permanente Analyse führt deshalb zur Paralyse", wie es ein Befragter ausdrückt. Von der Auflösung der UdSSR im Jahre 1991 über die Finanzkrise im August 1998 bis zur Wahl Putins im März 2000 sind in Rußland so viele überraschende und von niemandem vorhergesagten Ereignisse geschehen, daß es illusorisch wäre anzunehmen, relevante Trends frühzeitig und zuverlässig erkennen zu können. Entscheidend ist vielmehr eine flexible, gleichzeitig aber auch verläßliche und kontinuierliche Unternehmungspolitik, um das Interesse an einer strategischen Entwicklung des Engagements in Rußland deutlich zu machen.

\section{Instrumente des Public Affairs Management in Tochtergesell- schaften deutscher Multinationaler Unternehmungen in Rußland}

Dem zugrundeliegenden Bezugsrahmen entsprechend wurde neben der Bedeutung sozio-politischer Anliegen und Interessengruppen auch der Einsatz unterschiedlicher Instrumente des Public Affairs Management untersucht (vgl. Abb. 3). Die Einschätzungen der Befragten werden im folgenden ausführlich dargestellt und kritisch analysiert.

\subsection{Lobbying}

Alle Befragte messen engen persönlichen Beziehungen $\mathrm{zu}$ Entscheidungsträgern in der zentralen, regionalen und lokalen Administration eine große Bedeutung für Ihre Tätigkeit zu. Diese dienen nicht nur dazu, die Orientierung in einer als ausgesprochen komplex und dynamisch empfundenen Umwelt zu erleichtern, sondern auch der Durchsetzung konkreter unternehmungspolitischer Interessen. Entsprechend kommt dem Lobbying in allen untersuchten Unternehmungen im Vergleich zu anderen Instrumenten des Public Affairs Management die größte Bedeutung zu.

Im Unterschied $\mathrm{zu}$ den USA, wo die Tätigkeit von Lobbyisten umfassend gesetzlich geregelt ist und alle gesellschaftlich relevanten Gruppen etwa in Form von Hearings auf den politischen Meinungsbildungsprozeß Einfluß nehmen können, sind diese Einflußmöglichkeiten in Rußland jedoch weitestgehend auf die Leiter großer Unternehmungen und Banken beschränkt. Deren Einflußnahme geschieht zudem weniger durch Informationen oder die Mitwirkung in Fachausschüssen und Expertenkomissionen, als vielmehr durch die massive finanzielle Unterstützung bestimmter politischer Gruppen und die Beeinflussung der öffentlichen Meinung durch eigene Zeitungen und 
Fernsehsender (vgl. dazu ausführlich Šamchalov 2000, S. 198 ff.). ${ }^{8}$ Vor allem aber setzt Lobbying in Rußland nicht während des Gesetzgebungsverfahrens, sondern am konkreten Einzelfall an. Die Folge davon ist eine Vielzahl von undurchschaubaren Ausnahmeregelungen, die immer nur für eine bestimmte Unternehmung gelten. Während man in den USA deshalb von einem institutionalisierten ex-ante Lobbying sprechen könnte, ist die Situation in Rußland eher durch ein ad-hoc betriebenes ex-post Lobbying gekennzeichnet.

\section{Abb. 3: $\quad$ Bedeutung von Instrumenten des Public Affairs-Management}

Die Lobbyingaktivitäten der untersuchten Unternehmungen erfolgen deshalb

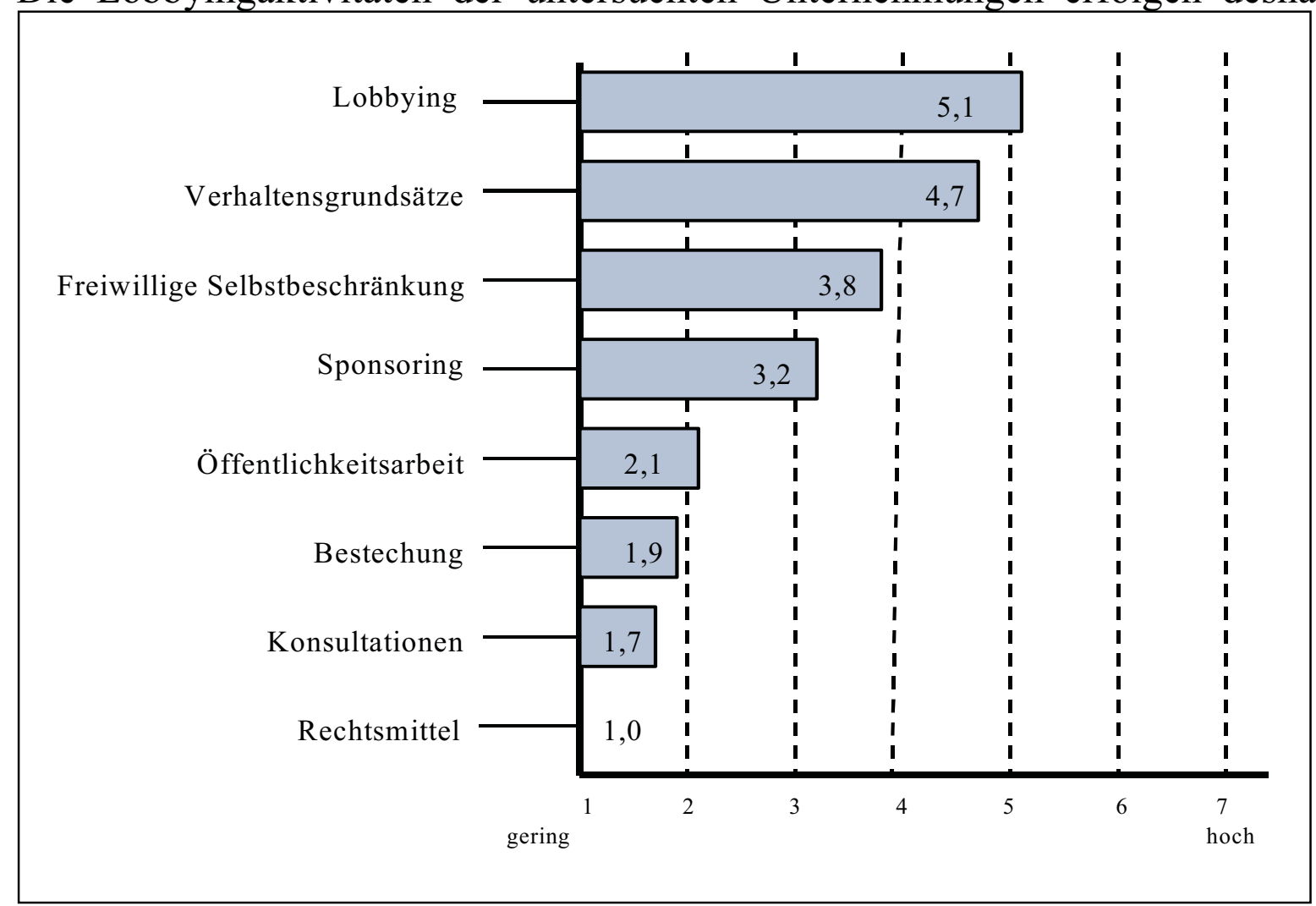

nicht in Form transparenter, institutionalisierter Strukturen, sondern vielmehr durch die fallweise Nutzung privater und geschäftlicher Kontakte. Zum Aufbau dieser Kontakte dienen etwa Empfänge der Botschaften oder Veranstaltungen von Interessenverbänden ausländischer Unternehmungen. Nach Auskunft der Befragten ist der Aufbau von Beziehungen zu wichtigen Entscheidungsträgern ein sehr langwieriger Prozeß, der zumeist einen mehrjährigen Aufenthalt in Rußland erfordert. Auffallend ist auch die relativ hohe Zahl von Mitarbeitern mit Adelstiteln sowie von ehemaligen Politikern und Diplomaten, die in diesem Bereich für ausländische Unternehmungen tätig sind. So wird etwa die Repräsentanz von DaimlerChrysler in Moskau durch Andreas Meyer-Landrut, den

${ }^{8}$ Eine besondere Bedeutung spielen in diesem Zusammenhang die sog. Oligarchen, auf die im nächsten Abschnitt ausführlich eingegangen werden wird. 
ehemaligen deutschen Botschafter in Rußland, geleitet (vgl. DaimlerChrysler 2000).

\subsection{Bestechung}

Von den Befragten wurde übereinstimmend das Ausmaß der Korruption in Rußland als sehr hoch bezeichnet. Auf einer Likertskala mit den Werten $1=$ keine Bedeutung und $7=$ sehr große Bedeutung liegt der Mittelwert der Einschätzungen der Befragten bei 5,0.

Die weite Verbreitung der Korruption in Rußland wird vielfach auf die enorme Größe des Landes zurückgeführt. Bereits im zaristischen Rußland erschwerte diese eine effiziente Verwaltung des Landes, wodurch wiederum das Vertrauen in die staatliche Administration nur gering war. ${ }^{9}$ Die Rechtsordnung war zudem stets ein Obrigkeitssystem, das durch autokratische Macht bzw. die kommunistische Ideologie und nicht durch demokratische Verfahren legitimiert war. Verstärkt wurde diese Legitimationsschwäche des Staates durch das sehr niedrige Einkommen der russischen Staatsbediensteten, das diese geradezu dazu zwang, sich nach lukrativen Nebenerwerbsquellen umzusehen (vgl. Holmes 1993, S. 38).

Seit dem Zusammenbruch der UdSSR und der Einleitung des Transformationsprozesses der russischen Wirtschaft in eine Marktwirtschaft stellen vor allem das niedrige Einkommensniveau der Staatsbediensteten und deren abnehmende Arbeitsplatzsicherheit im Zuge häufiger Regierungsumbildungen die zentralen Ursachen für eine ausgeprägte Korruption dar. Zwar herrscht empirischen Untersuchungen zufolge bei den meisten Menschen ein ausgeprägtes Unrechtsbewußtsein vor, in der Praxis gilt jedoch die Einstellung: "(It is) foolish to give (but) yet more foolish not to take" (Grødeland/Koshechkina/Miller 1998).

Diese Einstellung hat wesentlich dazu beigetragen, daß die 1991 eingeleitete Privatisierung des Staatseigentums unfair und in weiten Bereichen rechtswidrig verlaufen ist. Obwohl das russische Privatisierungsprogramm mit dem sog. Vouchersystem sozialen Kriterien eine große Bedeutung beigemessen hat, ist gegenwärtig eine sehr starke Vermögenskonzentration zu beobachten. Profitiert haben davon vor allem ehemalige Direktoren von Staatsbetrieben, höhere Staatsbedienstete und führende Funktionäre der KPdSU, da sie entweder im Besitz wertvoller materieller oder struktureller Ressourcen waren oder über direkte Kontakte zu zentralen Entscheidungsträgern verfügten.

9 "Der Zar ist weit, und Gott ist im Himmel" lautet ein bekanntes russisches Sprichwort, das diesen Sachverhalt anschaulich illustriert. 
Dies gilt in besonderem Maße für die sog. Oligarchen. ${ }^{10}$ Mit diesem durch den ehemaligen stellvertretenden Ministerpräsidenten Boris Nemcov geprägten Begriff werden die häufig aus der ehemaligen sowjetischen nomenklatura hervorgegangenen Leiter russischer Großunternehmungen und Finanzholdings bezeichnet, die während der Amtszeit von Präsident Jelzin große Vermögen angehäuft und etwa durch die Finanzierung von Wahlkämpfen oder die Kontrolle wichtiger Medien auch großen Einfluß auf die russische Politik hatten (vgl. Schröder 1999; Pappé 2000). Deren Methoden überschritten dabei auch nach deren eigenem Bekunden häufig die Grenzen der Legalität. So hat etwa Boris Berezovskij kurz nach der Wahl Vladimir Putins eine Amnestie für alle russischen Unternehmer gefordert, "da in den letzten zehn Jahren nur derjenige in Rußland nicht gegen Gesetze verstoßen hat, der untätig geblieben ist". Die Korruption wurde so zu einer "neunköpfigen Hydra" (Schüsselbauer 1999, S. 279), die alle Bereiche von Wirtschaft und Politik durchdringt.

Entgegen dieser auch durch die Befragten bestätigten weiten Verbreitung der Korruption ist diese nach deren Auskunft für die untersuchten Unternehmungen nur von sehr geringer Relevanz (vgl. Abb. 3). Diese Einschätzung erscheint jedoch zweifelhaft. So berichten viele Befragte relativ offen über "Transaktionskosten" und "betriebsbedingte Aufwendungen", die von der Überreichung von "Souvenirs" an russische Zöllner zur Beschleunigung der Grenzabfertigung und Einladungen wichtiger Entscheidungsträger $\mathrm{zu}$ "Geschäftsreisen" nach Deutschland bis zur kostenlosen Zurverfügungstellung von Produkten der jeweiligen Unternehmung zur "Überprüfung der versprochenen Qualität" reichen. Diese nicht-monetären Formen der Vorteilsgewährung werden jedoch nicht als Bestechung aufgefaßt, da sie in Rußland zum normalen Geschäftsgebaren zählten und zudem auch in anderen Ländern üblich seien.

Übereinstimmend wurde dagegen auf die Gefahr hingewiesen, sich durch die Zahlung von Bestechungsgeldern etwa zur Erlangung von Aufträgen erpreßbar und damit abhängig zu machen. Dies gilt in besonderem Maße für den Umgang mit den Oligarchen, mit denen die Mehrheit der Befragten deshalb geschäftliche Kontakte explizit vermeidet. Diese Form der freiwilligen Selbstbeschränkung bleibt jedoch in ihrer faktischen Bedeutung fragwürdig, wie die Äußerung eines Befragten verdeutlicht: "Von denjenigen, die in den letzen 10 Jahren reich geworden sind, hat keiner sein Geld auf ehrliche Art und Weise verdient. Anders kann man in Rußland gar keine Geschäfte machen. Wir arbeiten jedoch nicht direkt mit diesen Personen, sondern mit Unternehmungen zusammen. Mit den Oligarchen haben wir gar nichts zu tun. Diese stehen eher im Hintergrund."

${ }^{10}$ Dazu zählen etwa Vagit Alekperov (LUKojl), Boris Berezovskij (ORT), Anatolij_ubajs (ÉES Rußland), Michail Fridman (Al'fa-Gruppe), Vladimir Gusinskij (Most), Vladimir Potanin (Interros-Gruppe) und Rem Vjachirev (Gazprom). 
Bezeichnend ist auch die von der Mehrheit der Befragten gemachte Aussage "Es gibt viele andere ausländische Unternehmungen, die sich den hiesigen Spielregeln angepaßt haben. Wir haben jedoch einen guten Ruf zu verlieren und halten uns strikt an Recht und Gesetz." Daran knüpft sich einerseits die Frage, was als "Recht und Gesetz" verstanden wird, wenn gleichzeitig auf die großen kulturellen Unterschiede zwischen Deutschland und Rußland hingewiesen wird, die berücksichtigt werden müßten, um in Rußland erfolgreich tätig sein zu können. Offensichtlich existiert in Rußland eine beträchtliche Grauzone, innerhalb derer nicht-monetäre Vorteilsgewährungen erwartet und nicht negativ sanktioniert werden. Andererseits ist die Begründung für den behaupteten Verzicht auf den Einsatz von Bestechungsgeldern auffallend. Dieser wird nicht mit eigenen moralischen Überzeugungen erklärt, sondern mit der Angst vor länderübergreifenden Imageverlusten, die langfristig auch zu negativen Auswirkungen auf den Shareholder Value führen könnten. Diese bei der Mehrheit der Befragten vorherrschende instrumentelle Ethik wird auch durch eine nähere Analyse der in den untersuchten Unternehmungen geltenden Verhaltensgrundsätze bestätigt.

\subsection{Verhaltensgrundsätze}

Zwei Drittel der untersuchten Unternehmungen haben explizite Verhaltensgrundsätze bzw. Ethik-Kodizes für ihre Tätigkeit aufgestellt. Diese sollen als oberste Richtlinien das Handeln der Mitarbeiter leiten. In vielen Fällen gelten die Verhaltensgrundsätze auch für das Verhalten der Mitarbeiter im privaten Bereich. So wird diesen ein sensibles und zurückhaltendes Auftreten in der Öffentlichkeit empfohlen, das etwa die provozierende Zurschaustellung westlicher Luxusgüter vermeidet.

Auffallend ist, daß die Verhaltensgrundsätze in keiner Unternehmung speziell für Rußland entwickelt, sondern von der deutschen Muttergesellschaft übernommen wurden. Lediglich in etwa einem Viertel der Fälle fand eine geringfügige Anpassung an die spezifischen russischen Bedingungen statt. In einer Unternehmung wurden die ansonsten weltweit gültigen Verhaltensgrundsätze für Rußland explizit außer Kraft gesetzt. Dieses Ergebnis steht in einem deutlichen Kontrast $\mathrm{zu}$ einer Untersuchung unter Tochtergesellschaften deutscher Unternehmungen in Indien, wonach in der Hälfte der untersuchten Unternehmungen die dort geltenden Verhaltensgrundsätze speziell für die indische Tochtergesellschaft entwickelt wurden, um explizit dem in der Muttergesellschaft dominierenden Shareholder Value-Denken entgegenzuwirken (vgl. Berg/Holtbrügge 2001).

Auch in Rußland sind sich die meisten Befragten zwar der moralischen Aspekte ihrer Tätigkeit bewußt, diese werden jedoch weniger offen diskutiert, als vielmehr an die Muttergesellschaft oder die russischen Geschäftspartner "outgesourced", wie ein Befragter anschaulich darlegt: "Wir arbeiten nur mit 
russischen Unternehmungen zusammen, die sich als vertrauenswürdig erwiesen haben. Was diese mit unseren Produkten machen und wie sie diese weiter vertreiben, wissen wir nicht und wollen es auch nicht wissen." Typisch ist auch die Aussage eines anderen Befragten, wonach etwa die Lagerung gefährlicher Produkte bei vielen Lieferanten und Kunden grob fahrlässig ist, die Unternehmung darauf aber letztlich trotz vielfältiger Appelle an die Einhaltung der Sicherheitsbestimmungen keinen Einfluß habe: "Bislang haben wir einfach Glück gehabt."

Insgesamt lassen die Aussagen der Befragten den Schluß zu, daß in Tochtergesellschaften deutscher Unternehmungen in Rußland nur in einem sehr begrenzten Maße eine Auseinandersetzung mit ethischen Fragen der Unternehmungsführung stattfindet. Auch die Frage nach unterschiedlichen ethischen Normen und Werten in Deutschland und Rußland wurde deshalb überwiegend mit dem Verweis auf Besonderheiten der russischen Landeskultur wie die Bedeutung persönlicher Beziehungen und spezielle "Trinksitten" beantwortet. Im Mittelpunkt der Aufmerksamkeit stehen damit weniger moralische und normative Grundsatzfragen, als vielmehr der Umgang mit unterschiedlichen Sitten und Gebräuchen. Es geht weniger um Ethik, als um Etikette.

\subsection{Freiwillige Selbstbeschränkung}

Eng verbunden mit der Aufstellung von Verhaltensgrundsätzen ist die freiwillige Selbstbeschränkung von Unternehmungen. Diese können entweder dazu dienen, sich durch den Verzicht auf die Durchsetzung eines gesetzlich verankerten Rechts oder die Ablehnung von als unmoralisch empfundenen nationalen Geschäftspraktiken einen Vertrauensvorschuß bei wichtigen soziopolitischen Interessengruppen $\mathrm{zu}$ verschaffen, um dadurch andere unternehmungspolitische Ziele leichter durchsetzen zu können, oder um bestimmte moralische Überzeugungen sichtbar zum Ausdruck zu bringen (vgl. Welge/Holtbrügge 1998, S. 277 f.).

Ein weit verbreitetes und von der Mehrheit der Russen nicht als unmoralisch empfundenes Verhalten ist etwa die Umgehung von Gesetzen, die als unsinnig oder einseitig aufgefaßt werden (vgl. dazu ausführlich Puffer/McCarthy 1998, S. $430 \mathrm{ff}$.). Dies gilt insbesondere für die Steuergesetzgebung. So zitiert etwa Utkin (2000, S. 130) einen führenden Mitarbeiter einer russischen Bank: "Warum jemand keine Steuern zahlt oder versucht, keine zu zahlen, ist verständlich. Es ist schwer zu erklären, warum andere den Gesetzen treu sind". Selbst dem ehemaligen Präsidenten Boris Jelzin wird die Aussage zugeschrieben: "Unternehmer, die alle geforderten Steuern zahlen, werden als Geschäftsleute nicht überleben" (zit. nach Utkin 2000, S. 130).

Die Befragten weisen demgegenüber übereinstimmend darauf hin, daß sie die von ihren Geschäftspartnern häufig vorgeschlagenen "Steuersparmodelle" grundsätzlich ablehnen und die russischen Gesetze - auch wenn deren Umgehung in bestimmten Situationen allgemein üblich ist und weitgehend ak- 
zeptiert wird - strikt einhalten. In einigen Fällen ging das bereits zu Lasten möglicher Geschäfte. Auch andere Rechtsnormen etwa in den Bereichen des Umweltschutzes und der Arbeits- und Entlohnungsbedingungen werden nicht nur erfüllt, sondern deren Mindestbestimmungen teilweise deutlich überschritten.

Zur Begründung ihrer Haltung geben die Befragten nahezu übereinstimmend an, daß es sich bei dem Engagement ihrer Unternehmung in Rußland um ein strategisches Investment handelt und die Muttergesellschaft der Verfolgung gesamtunternehmungsbezogener Ziele eine höhere Priorität einräumt als kurzfristigen Gewinnen. Ähnlich wie bei der Aufstellung von Verhaltsgrundsätzen ist die in bestimmten Bereichen vollzogene freiwillige Selbstbeschränkung damit überwiegend nicht auf ethische Überzeugungen, sondern auf strategische und landesübergreifende Überlegungen der Unternehmungen zurückzuführen. Besonders anschaulich und aufrichtig schildert dies einer der Befragten: "Die Frage, ob ein Engagement in Rußland überhaupt moralisch zu vertreten ist, muß man sich angesichts der Ereignisse in Tschetschenien immer wieder stellen. Andererseits ist es für ausländische Unternehmungen gegenwärtig relativ leicht, sich eine kritische Haltung zu leisten, auch wenn dadurch Geschäfte verlorengehen. Bei der derzeitigen Wirtschaftslage in Rußland ist das zu verschmerzen. Wenn es in Rußland aber auch einmal Wachstumsraten von 8 Prozent jährlich wie in China geben wird, ist es fraglich, wie viele Unternehmungen sich noch ein moralisches Gewissen leisten werden."

\subsection{Sponsoring}

Viele Befragte verweisen im Zusammenhang mit ihrer ethischen Verantwortung gegenüber Rußland auf die Sponsoringaktivitäten, die ihre Unternehmung in diesem Land durchführt. Mit einer Ausnahme sind alle untersuchten Unternehmungen in diesem Bereich tätig. Im Vordergrund steht dabei häufig das Stipendienprogramm für russische Führungsnachwuchskräfte zum Studium in Deutschland, das auf Initiative des deutschen Botschafters in Rußland eingerichtet worden ist, von insgesamt 33 deutschen Unternehmungen in Rußland finanziell gefördert und durch den DAAD organisatorisch betreut wird. ${ }^{11}$ Weitere Schwerpunkte sind das Sponsoring von Kultur- und Sportveranstaltungen sowie von sozialen und kirchlichen Einrichtungen. Viele deutsche Unternehmungen beteiligen sich darüber hinaus an den sozialen und kulturellen Aktivitäten des Verbands der deutschen Wirtschaft in der Russischen Föderation.

Die Motive, die die untersuchten Unternehmungen mit diesem Sponsoringaktivitäten verbinden, sind unterschiedlich. Während $31 \%$ primär den damit verbundenen Imagegewinn für ihre Unternehmung herausstellen, 
steht für 54\% der Befragten die historische und gesellschaftliche Verantwortung gegenüber Rußland im Vordergrund. Deutliche Unterschiede zeigen sich auch bezüglich der Institutionalisierung von Sponsoringaktivitäten. Während in der Hälfte der Unternehmungen über deren Umfang und Inhalt ad-hoc durch den Leiter der Unternehmung entschieden wird, existiert in der anderen Hälfe der Unternehmungen eine detaillierte Sponsoring-Strategie, die zudem weitgehend mit der Muttergesellschaft abgestimmt ist. Zu dem Umfang ihrer Sponsoringaktivitäten konnten oder wollten nur drei Unternehmungen konkrete Angaben machen. Die dabei genannten Budgets von 10.000 DM, 20.000 DM bzw. 200.000 DM pro Jahr erscheinen sowohl absolut als auch im Verhältnis zu den von diesen Unternehmungen in Rußland erzielten Umsätzen sehr gering.

\section{6 Öffentlichkeitsarbeit}

Alle untersuchten Unternehmungen zeichnen sich durch eine sehr restriktive und selektive Öffentlichkeitsarbeit aus, die bereits deren Standortwahl kennzeichnet. So befinden sich die meisten Unternehmungen in relativ unscheinbaren Gebäuden in Außenbezirken von Moskau. Vielfach ist an den Gebäuden nicht einmal ein Firmenschild angebracht. Auch gegenüber den Medien verhalten sich die untersuchten Unternehmungen sehr zurückhaltend. Informationen werden zumeist nur an ausgewählte Journalisten weitergegeben, die sich als seriös und objektiv erwiesen haben.

Als zentralen Grund für dieses sehr diskrete Auftreten in der Öffentlichkeit, das häufig in starkem Kontrast zu dem Verhalten der Unternehmungen in anderen Ländern steht, führen die Befragten die Angst vor unliebsamen Kontakten mit der Mafia an. Viele ausländische Unternehmungen präferieren deshalb auch die Errichtung von Repräsentanzen, die de iure lediglich als Kontaktstelle für die deutsche Muttergesellschaft agieren und keine eigenen finanziellen Transaktionen durchführen dürfen. Lediglich drei Befragte äußerten die Auffassung, $\mathrm{da} ß$ eine solche Zurückhaltung angesichts der nachlassenden Bedrohung durch kriminelle Strukturen nicht länger notwendig sei. Bei diesen Unternehmungen ist deshalb gegenwärtig eine Neuorientierung der Öffentlichkeitsarbeit erkennbar, die sich u.a. im Umzug in neu errichtete Gebäude im Stadtzentrum von Moskau oder der Einrichtung einer Abteilung für Öffentlichkeitsarbeit niederschlägt.

\subsection{Konsultationen}

Ein wichtiges Instrument zur Berücksichtigung sozio-politischer Anliegen bei unternehmungspolitischen Entscheidungen ist die Konsultation wichtiger Interessengruppen. Da gesellschaftliche und ökologische Interessengruppen in Rußland im Vergleich zu politischen Interessengruppen nur einen sehr geringen Einfluß besitzen, finden regelmäßige nicht-institutionalisierte Konsultationen jedoch lediglich in drei der untersuchten Unternehmungen statt. Deren 
Bedeutung wird zudem im Vergleich $\mathrm{zu}$ anderen Instrumenten des Public Affairs Management nur als gering eingeschätzt. Die Beteiligung von Interessengruppen in einem Beirat oder Kuratorium wird in keiner Unternehmung praktiziert.

\subsection{Rechtsmittel}

Auch Rechtsmittel werden von den Befragten übereinstimmend als unbrauchbares Instrument zur Durchsetzung von Interessen angesehen. Obwohl in den letzten Jahren die Zahl der Klagen ausländischer Unternehmungen vor dem Arbitragegericht zugenommen hat (vgl. Nešataeva 1998), präferieren die Unternehmungen weiterhin außergerichtliche Verfahren der Konflikthandhabung. Zwar wird der russischen Gerichtsbarkeit überwiegend bescheinigt, ausländische Unternehmungen nicht zu diskriminieren, deren Verfahren werden jedoch als ausgesprochen aufwendig und langwierig bezeichnet. Zudem ist es in der Vergangenheit häufig vorgekommen, daß sich der vor Gericht erstrittene Rechtstitel nicht in faktisches Recht umsetzen ließ. "Man bekommt zwar moralisch Recht, aber kein Geld", so einer der Befragten.

\section{Zusammenfassung und Schlußfolgerungen}

Das Ziel der vorliegenden Untersuchung bestand darin zu untersuchen, wie das Verhalten deutscher Unternehmungen in Rußland durch unterschiedliche soziopolitische Interessengruppen beeinflußt wird und welche Strategien, Instrumente und Organisationsformen des Public Affairs Management sie verfolgen, um mit den von ihren Interessengruppen erhobenen Ansprüchen umzugehen. Die Befragung der höchstrangigen Stammhausdelegierten von 14 Tochtergesellschaften deutscher Multinationaler Unternehmungen machte deutlich, daß die untersuchten Unternehmungen primär mit politischen Interessengruppen konfrontiert sind. Die größte Bedeutung besitzt die russische Regierung, gefolgt von der jeweiligen regionalen und lokalen Administration. Dieses Ergebnis unterstreicht, daß Regierung und Verwaltung trotz des Anfang der neunziger Jahre eingeleiteten wirtschaftlichen Transformationsprozesses weiterhin eine große Bedeutung für ausländische Unternehmungen besitzen und sich sowohl restriktiv als auch unterstützend auf deren unternehmungspolitische Entscheidungen auswirken können. Von den Befragten werden deshalb übereinstimmend enge persönliche Kontakte zu wichtigen Entscheidungsträgern als zentrale Erfolgsvoraussetzung einer erfolgreichen Tätigkeit in Rußland angesehen.

Im Vergleich zu den politischen Interessengruppen kommt der Mafia, wichtigen Verbänden und den Medien eine deutlich geringere Bedeutung zu, während Gewerkschaften, Bürgerinitiativen und NGOs keinen nennenswerten Einfluß auf die Tätigkeit ausländischer Unternehmungen ausüben. Gleiches gilt für internationale Organisationen. Obwohl Rußland gegenwärtig mit einer Vielzahl so- 
zialer und ökologischer Probleme konfrontiert ist, sind diese für ausländische Unternehmungen weitgehend irrelevant, da es keine Interessengruppen gibt, die diese Anliegen einflußreich vertreten. Im Vordergrund stehen vielmehr politische und ökonomische Interessen, die den Erhalt, die Sicherung und den Ausbau von Macht beinhalten. Auffallend ist zudem, daß die untersuchten Unternehmungen nur mit wenigen zentralen Interessengruppen konfrontiert sind, d.h. die Zahl der relevanten Stakeholder gering ist.

Ein weiteres zentrales Ergebnis der Untersuchung ist, daß sich auch die für ausländische Unternehmungen relevanten sozio-politischen Interessengruppen weitgehend passiv verhalten. Dies bedeutet, daß primär nicht die Interessengruppen Anliegen an die Unternehmungen herantragen, sondern die Unternehmungen bestimmte Interessen verfolgen, die sie nur mit der Hilfe bestimmter sozio-politischer Interessengruppen umsetzen können. Einige gesellschaftliche Gruppen wie die Mafia, Geheimdienste oder Medien können zudem eher als potentielle oder latente Interessengruppen bezeichnet werden. Ihr Einfluß auf die Unternehmungspolitik besteht weniger in direkten und zielgerichteten Aktionen als in dem Bemühen der Unternehmungen, Kontakte mit diesen Gruppen weitgehend zu vermeiden.

Die Analyse der von den untersuchten Unternehmungen eingesetzten Instrumente des Public Affairs Management unterstreicht die große Bedeutung politischer Interessengruppen, die sich in ausgeprägten Lobbyingaktivitäten niederschlägt. Darüber hinaus kommt der Aufstellung von Verhaltensgrundsätzen und der freiwilligen Selbstbeschränkung eine relativ große Bedeutung zu. Mit einer Ausnahme sind darüber hinaus alle Unternehmungen im Bereich des Sponsoring aktiv, während die übrigen Instrumente des Public Affairs Management nicht eingesetzt werden.

Die zwischen den untersuchten Unternehmungen $\mathrm{zu}$ beobachtenden Unterschiede können insbesondere auf personelle Faktoren zurückgeführt werden. Aufgrund der relativ geringen Unternehmungsgröße hängen die Aktivitäten im Bereich des Public Affairs Management vor allem vom Alter der entsandten Stammhausdelegierten und deren Einstellung gegenüber dem Gastland ab. Ältere Mitarbeiter mit einer hohen, häufig durch familiäre Beziehungen geprägte Affinität zu Rußland zeichnen sich zumeist durch umfassende Kenntnisse der russischen Geschichte und Kultur aus, die sich in einer ausgeprägten Auseinandersetzung mit ethischen Grundsatzfragen niederschlägt. Bei älteren Mitarbeitern, die ihre Entsendung eher als "Strafversetzung" empfinden, ist dagegen zwar eine gewisse Sensibilität gegenüber den in Rußland vorherrschenden politischen, sozialen und ökologischen Zuständen spürbar. Diese hat jedoch keinen Einfluß auf ihre unternehmungspolitischen Entscheidungen. Kennzeichnend ist etwa die Aussage eines Befragten: "In ein paar Jahren werde ich pensioniert, da will ich mir keine Feinde mehr machen." Bei jüngeren Mitarbeitern steht schließlich die eigene Karriere im Vordergrund. 
Die Haltung gegenüber dem Gastland ist deshalb eher indifferent, und die eingesetzten Instrumente des Public Affairs Management vor allem auf einen hohen ökonomischen Erfolg ausgerichtet. Gesellschaftliche Aspekte werden für die eigene Tätigkeit als irrelevant und politische Aspekte nur insofern als bedeutsam angesehen, als sie die eigenen unternehmungspolitischen und persönlichen Ziele unmittelbar berühren.

\section{Literatur}

Apressyan, R.G.: Business Ethics in Russia. In: Journal of Business Ethics, Vol. 16, 1997, S. 1561-1570.

Bell, C.: Staat, Föderalismus, Transformation: Theorie und Rußländische Realität. In: Spontaner oder gestalteter Prozeß? Die Rolle des Staates in der Wirtschaftstransformation osteuropäischer Länder, hrsg. v. H.-H. Höhmann. Baden-Baden 1999, S. 272-296.

Berg, N./Holtbrügge, D.: Zwischen Markt und Moral. Ökonomische, politische und ethische Aspekte der Tätigkeit ausländischer Unternehmungen in der VR China. Dortmunder Beiträge zur Unternehmungsführung, Nr. 30, Dortmund 2000.

Berg, N./Holtbrügge, D.: Public Affairs Management Activities of German Multinational Corporations in India. In: Journal of Business Ethics, Vol. 20, 2001 (im Druck).

DaimlerChrysler: Koncern “DajmlerKrajsler” v Rossii. Moskau 2000.

Elder, A.: Roubles to Dollars. Making Money on Russia's Exploding Financial Frontier. New York et al. 1999.

Freeman, R.E.: Strategic Management: A Stakeholder Approach. Boston 1984.

Glucksmann, A.: Stalin dachte nicht anders. Der Krieg in Tschetschenien vernichtet die moralischen Fundamente der russischen Gesellschaft. In: Die Zeit, 27.12.2000, S. 11.

Götz, R./Halbach, U.: Politisches Lexikon Rußland. Die nationalen Republiken und Gebietseinheiten der Rußländischen Föderation. München 1994.

Grødeland, Å. B./Koshechkina, T.Y./Miller, W.L.: 'Foolish to Give and Yet More Foolish Not to Take' - In-depth Interviews with Post-Communist Citizens on Their Everyday Use of Bribes and Contacts. In: Europe-Asia Studies, Vol. 50, H. 4, 1998, S. 651-677.

Holmes, L.: The End of Communist Power: Anti-Corruption Campaigns and Legitimation Crisis. Cambridge 1993.

Holtbrügge, D.: Joint Ventures in der UdSSR: Privatkapitalistische Inseln oder intersystemare Unternehmungskooperationen? In: Aussenwirtschaft, 44. Jg., H. 3/4, 1989, S. 399-424.

Holtbrügge, D.: Personalmanagement multinationaler Unternehmungen in Osteuropa. Bedingungen - Gestaltung - Effizienz. Wiesbaden 1995.

Holtbrügge, D.: Erfolgsfaktoren ausländischer Direktinvestitionen in Rußland. In: Wirtschaftspartner Rußland. Rahmenbedingungen-Kooperationsstrategien-Erfahrungsberichte, hrsg. v. M.K. Welge u. D. Holtbrügge. Wiesbaden 1996, S. 19-43.

Kappeler, A.: Rußland als Vielvölkerreich. Entstehung, Geschichte, Zerfall. München 1992. 
Kostova, T./Zaheer, S.: Organizational Legitimacy Under Conditions of Complexity: The Case of the Multinational Enterprise. In: Academy of Management Review, Vol. 24, H. 1, 1999, S. 64-81.

Nešataeva, T.N.: Inostrannye predprinimateli v Rossii. Sudebno-arbitra_naja praktika. Moskau 1998.

Pappé, Ja.: Oligarchi._konomi_eskaja chronika, 1992-2000. Moskau 2000.

Puffer, S.M./McCarthy, D.J.: Business Ethics in a Transforming Economy: Applying the Integrative Social Contracts Theory to Russia. In: Ethics in International Management, hrsg. v. B.N. Kumar u. H. Steinmann. Berlin-New York 1998, S. 419-437.

Radaev, V.: Business Ethics in Russia: Contract Enforcement and Violence in the late 90s. Paper presented at the IV Chemnitz East Forum "Business Ethics in Central and Eastern Europe". Chemnitz, March 3-5, 1999.

Šamchalov, F.: Gosudarstvo i ékonomika. Osnovy vzaimodejstvija. Moskau 2000.

Šejnis, V.: Wie Rußland gewählt hat. Zum Fazit der Parlaments- und Präsidentschaftswahlen Dezember 1999/März 2000. In: Osteuropa, 50. Jg., 7, 2000, S. 759-778.

Schlott, W.: "Unterweltige" Rahmenbedingungen: Kriminalität, Mafia und Korruption in Rußland. In: Wirtschaftspartner Rußland. Rahmenbedingungen-Kooperationsstrategien-Erfahrungsberichte, hrsg. v. M.K. Welge u. D. Holtbrügge. Wiesbaden 1996, S. 159-177.

Schröder, H.-H.: El'tsin and the Oligarchs: The Role of Financial Groups in Russian Politics Between 1993 and July 1998. In: Europe-Asia Studies, Vol. 51, H. 6, 1999, S. 957988.

Schüsselbauer, G.: Korruption - ein grassierendes Phänomen in Mittel- und Osteuropa. In: Osteuropa-Wirtschaft, 44. Jg., H. 3, 1999, S. 276-283.

Seiffert, W.: Wladimir W. Putin. Wiedergeburt einer Weltmacht? München 2000.

Utkin, É.A.: Étika biznesa. Moskau 2000.

Welge, M.K./Berg, N.: Public Affairs-Management in multinationalen Unternehmungen - Ergebnisse einer empirischen Untersuchung deutscher Unternehmungen in Indien. In: Internationales Management. Auswirkungen globaler Veränderungen auf Wettbewerb, Unternehmensstrategie und Märkte, hrsg. v. J. Engelhard u. W.A. Oechsler. Wiesbaden 1999, S. 193-215.

Welge, M.K./Holtbrügge, D.: Internationales Management. Landsberg a.L. 1998. 